\title{
Syringomyelia associated with intradural arachnoid cysts
}

\author{
Langston T. Holly, M.D., AND UlRich Batzdorf, M.D. \\ Division of Neurosurgery, David Geffen School of Medicine, University of California at Los Angeles, \\ California
}

\begin{abstract}
Object. Intradural arachnoid cysts are relatively uncommon pouches of cerebrospinal fluid (CSF) found within the subarachnoid space. The authors present a series of eight symptomatic patients in whom syrinx cavities were associated with arachnoid cysts, and they discuss treatment strategies for this entity.

Methods. The population comprised eight men whose mean age was 50 years (range 35-81 years). All patients experienced gait difficulty, and it was the chief complaint in seven; arm pain was the primary complaint in one. No patient had a history of spinal trauma, meningitis, or previous spinal surgery at the level of the syrinx cavity or arachnoid cyst. In each patient imaging revealed a syrinx cavity affecting two to 10 vertebral levels. Posterior thoracic arachnoid cysts were found in proximity to the syrinx cavity in each case. There was no evidence of cavity enhancement, Chiari malformation, tethered cord, or hydrocephalus.

All patients underwent thoracic laminectomy and resection of the arachnoid cyst wall, and postoperative neurological improvement was documented in each case. The mean follow-up duration was 19 months (range 4-37 months). Follow-up magnetic resonance imaging demonstrated the disappearance of the arachnoid cyst and a significant decrease in syrinx cavity size in each patient.

Conclusions. Spinal arachnoid cysts can be associated with syringomyelia, likely due to alterations in normal CSF dynamics. In symptomatic patients these cysts should be resected and the normal CSF flow restored. The results of the present series indicate that neurological improvement and reduction in syrinx cavity size can be achieved in patients with syringomyelia associated with intradural arachnoid cysts.
\end{abstract}

\section{KEY WORDS • syringomyelia • arachnoid cyst • intradural lesion}

$\mathrm{S}$ YRINGOMYELIA is a complex disorder associated with a heterogeneous group of etiological entities including Chiari malformation, ${ }^{4}$ spinal trauma, ${ }^{6,8,9,13}$ and infection. ${ }^{5}$ Although quite diverse, all of these conditions share one common characteristic: an alteration in the normal CSF dynamics in the spinal subarachnoid space. The exact pathophysiological mechanism involved with syrinx cavity formation remains unknown, yet it is widely accepted that syringomyelia results from at least a partial obstruction of spinal CSF flow. Reestablishment of the normal CSF dynamics frequently results in reduction of the cavity size and neurological improvement.

Patients with spinal arachnoid cysts commonly present with neurological symptoms such as gait abnormalities or bladder dysfunction due to spinal cord compression. ${ }^{1,7,10}$, ${ }^{12,14}$ In a subset of cases arachnoid cysts can cause the formation of syrinx cavities, presumably due to subarachnoid

Abbreviations used in this paper: $\mathrm{CSF}=$ cerebrospinal fluid; $\mathrm{CT}=$ computed tomography; $\mathrm{MR}=$ magnetic resonance.
CSF flow obstruction, as with other causes of syringomyelia. The syrinx cavities can cause neurological sequelae that are independent, and yet these sequelae can also contribute to the effects of the arachnoid cyst. Spinal arachnoid cysts are relatively uncommon, and their association with syringomyelia has been mentioned infrequently in the literature. ${ }^{2,3,11,15}$

We provide an assessment of data obtained in a group of symptomatic patients with syringomyelia and an intradural arachnoid cyst in proximity to the syrinx cavity. The relationship between these pathological entities is discussed, and the diagnostic evaluation and management strategies are presented.

\section{Clinical Material and Methods}

\section{Patient Population}

The population was composed of eight symptomatic patients with an intradural arachnoid cyst and an adjacent syrinx cavity; the patients were treated at the University of 
TABLE 1

Summary of pre- and postoperative data obtained in eight patients with syringomyelia and arachnoid cysts

\begin{tabular}{|c|c|c|c|c|c|c|}
\hline \multirow[b]{2}{*}{ Case No. } & \multirow[b]{2}{*}{ Age (yrs), Sex } & \multicolumn{2}{|c|}{ Lesion Level } & \multirow[b]{2}{*}{ Chief Symptoms } & \multicolumn{2}{|c|}{ Syrinx Size $(\mathrm{mm})^{*}$} \\
\hline & & Arachnoid Cyst & Syrinx & & Preop & Postop \\
\hline 1 & $81, \mathrm{M}$ & $\mathrm{T} 3-4$ & T5-6 & gait difficulty & $20 \times 4$ & 0 \\
\hline 2 & $38, \mathrm{M}$ & $\mathrm{T} 7-8$ & $\mathrm{~T} 7-10$ & $\begin{array}{l}\text { gait difficulty, blad- } \\
\text { der incontinence }\end{array}$ & $60 \times 5$ & $60 \times 2$ \\
\hline 3 & $50, \mathrm{M}$ & $\mathrm{T}-5$ & $\mathrm{~T} 3-4$ & $\begin{array}{l}\text { gait difficulty, blad- } \\
\text { der incontinence }\end{array}$ & $20 \times 3$ & 0 \\
\hline 4 & $54, \mathrm{M}$ & T6-7 & $\mathrm{C} 2-\mathrm{T} 2$ & gait difficulty & $260 \times 5$ & $15 \times 1$ \\
\hline 5 & $60, \mathrm{M}$ & $\mathrm{T} 2-3$ & $\mathrm{C} 1-\mathrm{T} 1$ & $\begin{array}{l}\text { gait difficulty, arm } \\
\text { pain }\end{array}$ & $135 \times 10$ & $135 \times 2$ \\
\hline 6 & $42, \mathrm{M}$ & T5-6 & $\mathrm{C} 2-\mathrm{T} 3$ & gait difficulty & $160 \times 15$ & $140 \times 8$ \\
\hline 7 & $35, \mathrm{M}$ & T3-4 & $\mathrm{T} 2-3$ & gait difficulty & $31 \times 8$ & $8 \times 2$ \\
\hline 8 & $36, \mathrm{M}$ & T6-7 & $\mathrm{C} 4-\mathrm{T} 4$ & $\begin{array}{l}\text { gait difficulty, arm } \\
\text { pain }\end{array}$ & $130 \times 9$ & $90 \times 5$ \\
\hline
\end{tabular}

* Values reflect the length $\times$ the diameter of the syrinx.

California at Los Angeles Medical Center. We conducted a retrospective review of hospital chart and imaging data in all cases. The Office for Protection of Research Subjects at our institution approved the protocol for this study. The eight patients were all men whose mean age was 50 years (range $35-81$ years). The patient demographics are summarized in Table 1.

\section{Presenting Signs and Symptoms}

Gait difficulty, the most common presenting symptom, was seen in all eight patients. Six patients experienced abnormalities in lower-extremity sensation, and two suffered from bladder dysfunction. Upper-extremity neuropathic radicular pain was observed in two patients.

\section{Neurological Findings}

A diminished ability to perceive vibratory stimuli in the lower extremities was elicited in five patients, bilaterally in four and unilaterally in one. Three patients had symmetrical lower-extremity abnormalities in pain and temperature sensation. One patient experienced a decrease in toe proprioception in both feet. Three patients suffered abnormal upper-extremity sensation: an alteration in pain and temperature perception in two and a decreased vibratory sensation in one. Grade 4/5 lower-extremity strength was present in two patients; in one patient the weakness was only in dorsiflexion and in the other symmetrical weakness was observed in all muscle groups. Two patients were found to have asymmetrical bilateral intrinsic hand weakness. Hand motor function was Grades 3-/5 (left hand) and 4/5 (right hand) in one, and 5-/5 (left hand) and $4+/ 5$ (right hand) in the other. Four patients had at least one upper motor neuron sign in the lower extremities: hypertonia (three patients), hyperreflexia (three patients), clonus (two patients), and a Babinski sign (one patient).

\section{Medical History}

A history of spinal trauma or meningitis was absent in all cases. One patient had previously undergone spinal surgery, a T10-11 discectomy via a costotransversectomy. His arachnoid cyst was located at the T7-8 level, and the syrinx cavity terminated caudally at the T9-10 disc space.

\section{Imaging Findings}

Magnetic resonance imaging revealed thoracic syrinx cavities in each patient; the lesions ranged from two to 10 levels in length. The cavity extended into the cervical region in four patients. Posterior thoracic intradural arachnoid cysts were found in each patient in proximity to the syrinx cavities. In seven cases the arachnoid cysts were located caudal to the syrinx cavity and in one, cephalad to the cavity. The arachnoid cysts caused anterior spinal cord displacement (Figs. 1 and 2). There was no evidence of hydrocephalus, Chiari malformation, tethered cord, or syrinx cavity enhancement. In each case CT myelography confirmed the diagnosis of an arachnoid cyst. The CT scans were obtained approximately 30 minutes after administration of contrast medium. All of the arachnoid cysts immediately filled with the contrast agent, whereas the syrinx cavities did not.

\section{Surgical Management}

All eight patients underwent thoracic laminectomy, exposing the dura slightly rostral and caudal to the location of the arachnoid cyst. After completing the laminectomy, intraoperative ultrasonography was performed and provided information regarding cyst morphology, cyst size, and CSF flow dynamics (Fig. 2 right). The images were also quite helpful in determining the optimal length of the dural opening and preventing unnecessary neural exposure. The interface between the enlarged spinal cord harboring the syrinx cavity and the segment compressed by the cyst could be well visualized. The resolution of the ultrasound images allowed demonstration of the distinct CSF flow pulsations within the arachnoid cyst, syrinx cavity, and subarachnoid space. The pulsations within the subarachnoid space cephalad to the cyst appeared to be of normal magnitude, but the amplitude of the pulsations were markedly diminished caudal to the cyst. A similar observation was made after opening the dura. 

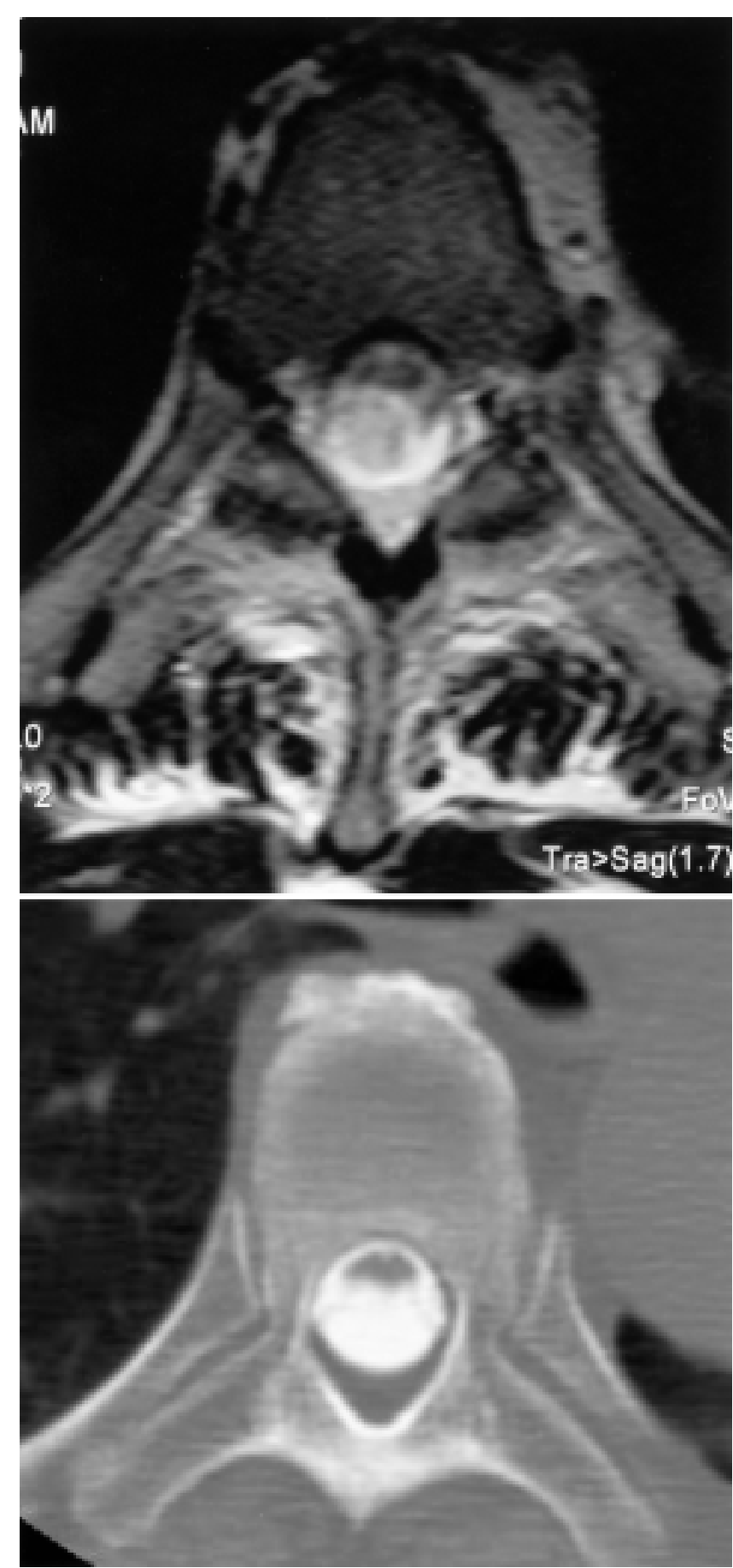

FIG. 1. Representative preoperative studies. Upper: Axial $\mathrm{T}_{2^{-}}$ weighted MR image obtained at T-6 demonstrating a posterior arachnoid cyst causing spinal cord compression. Lower: Postmyelography CT scan confirming the presence of the cyst; mass effect can be seen on the posterior portion of the spinal cord.

With the aid of the operating microscope, the arachnoid cyst was resected in each case, and normal CSF flow was reestablished. Although ultrasonographic examination was not routinely performed following cyst resection, we nonetheless confirmed intraoperatively that subarachnoid CSF pulsations had normalized. To augment the subarach- noid space reconstruction, a duraplasty was performed in two patients in whom the syrinx cavities were large. In six patients the dura mater was closed primarily. Intraoperatively the cysts appeared to have no unusual features, and pathological examination of the cyst wall showed normal arachnoid tissue.

\section{Results}

The mean follow-up period was 19 months (range 4-37 months). Neurological improvement was achieved in each case. All patients noted improvement in their ability to ambulate postoperatively. In both patients with radicular arm pain the symptom improved, and one of the two patients with bladder dysfunction noticed improvement postoperatively. In the two patients with preoperative lower-extremity weakness, motor function normalized following surgery. In the two patients with hand weakness, however, strength did not return postoperatively; in both of these patients significant preoperative atrophy of the intrinsic hand muscles had been documented. Three of the five patients with a lower-extremity vibratory sensation deficit noted improvement postoperatively, whereas in two the deficit persisted. One of three patients with altered lower-extremity pain/temperature sensation noted improvement, whereas dysfunction in the other two patients was unchanged. The patient with abnormal upper-extremity vibratory sensation experienced return of sensation postoperatively. Neither of the two patients with abnormal upper-extremity pain/temperature perception experienced any improvement after surgery.

Three-month postoperative MR imaging revealed complete excision of the arachnoid cysts and significantly diminished syrinx cavities in each case (Figs. 3 and 4; Table 1). There was one complication in the series. In one patient in whom an interscapular region incision was made, a superficial wound dehiscence developed several weeks postoperatively. This was likely due to physical activity that prematurely placed significant stress on the suture line. The wound was primarily reclosed and healed uneventfully.

\section{Discussion}

\section{Arachnoid Cysts}

Spinal arachnoid cysts are relatively uncommon lesions that can be found in the intra- or extradural compartments of the spinal canal. Like syringomyelia, a number of different theories have been proposed to explain the origin of arachnoid cysts, and the exact pathological mechanism of these lesions remains unclear. The true pathogenesis is likely to be multifactorial, although some of the better-established theories suggest a congenital cause. Perret, et al., ${ }^{12}$ have postulated that the cysts are caused by a dilatation of the septum posticum, a thin membranous arachnoid partition that longitudinally divides the posterior subarachnoid space in the midline. This theory satisfactorily explains the frequent occurrence of posterior arachnoid cysts but cannot account for arachnoid cysts that are found anterior to the spinal cord. Other authors have theorized that congenital dural defects allow the arachnoid to herniate through the dura via hydrostatic forces and lead to ex- 

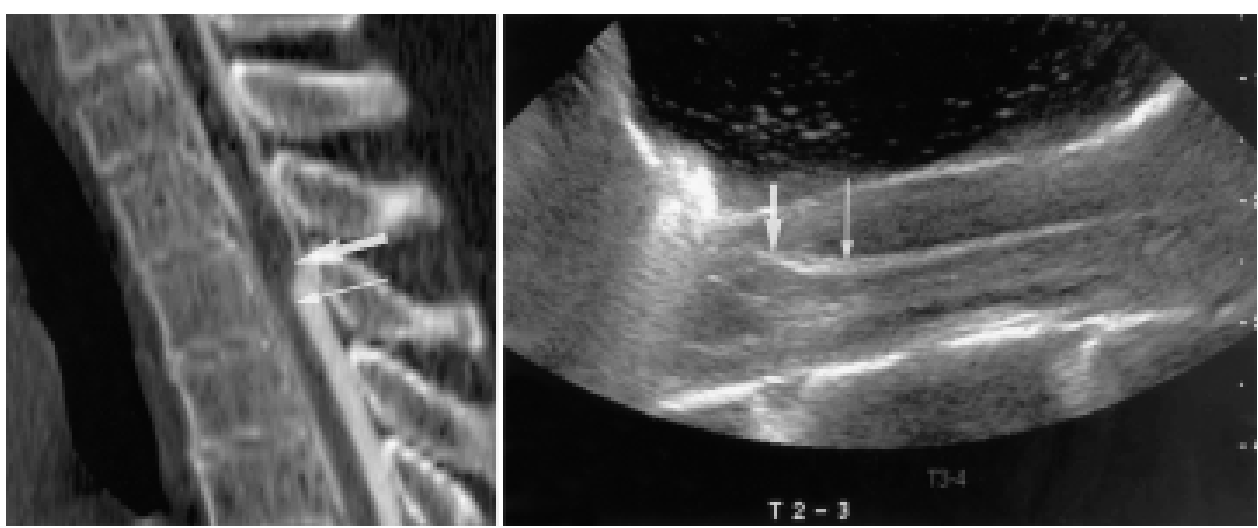

FIG. 2. Representative pre- (left) and intraoperative (right) imaging studies. Left: Sagittal postmyelography CT scan showing an area of spinal cord expansion (thick arrow) due to the syrinx cavity, as well as the abrupt change of spinal cord caliber (thin arrow) caused by a compressive posterior arachnoid cyst. Right: Intraoperative ultrasound image obtained in the same patient, revealing the dilated cord with the syrinx cavity (thick arrow) and narrowed spinal cord (thin arrow).

tradural cyst formation. ${ }^{1}$ Explanations of noncongenital causes include trauma, infection, subarachnoid hemorrhage, and previous surgery.

Spinal arachnoid cysts are most frequently found in the thoracic region, and intradural cysts are more common than extradural cysts. The most common presenting symptom is back pain emanating from the area in which the cyst is located. Direct spinal cord compression can cause neurological symptoms such as abnormalities in sensation, weakness, and bowel or bladder dysfunction. Arachnoid cysts may also cause alterations in CSF flow within the spinal subarachnoid space, leading to the formation of syrinx cavities. The actual incidence of syringomyelia in patients with arachnoid cysts is unknown.

\section{Imaging Studies}

Magnetic resonance imaging is the most commonly used modality for the detection of arachnoid cysts. Arachnoid cysts have the same imaging characteristics as CSF: hypointensity and hyperintensity on $\mathrm{T}_{1}$ - and $\mathrm{T}_{2}$-weighted MR images, respectively. In symptomatic patients these cysts can cause deformation of the neural elements, commonly flattening the spinal cord against the anterior portion of the spinal canal. Arachnoid cysts can also accompany a nerve root as it enters the neural foramen, leading to radicular-type symptoms. Aortic pulsation artifacts can cause the appearance of an abnormality that mimics the imaging characteristics of arachnoid cysts; however, this

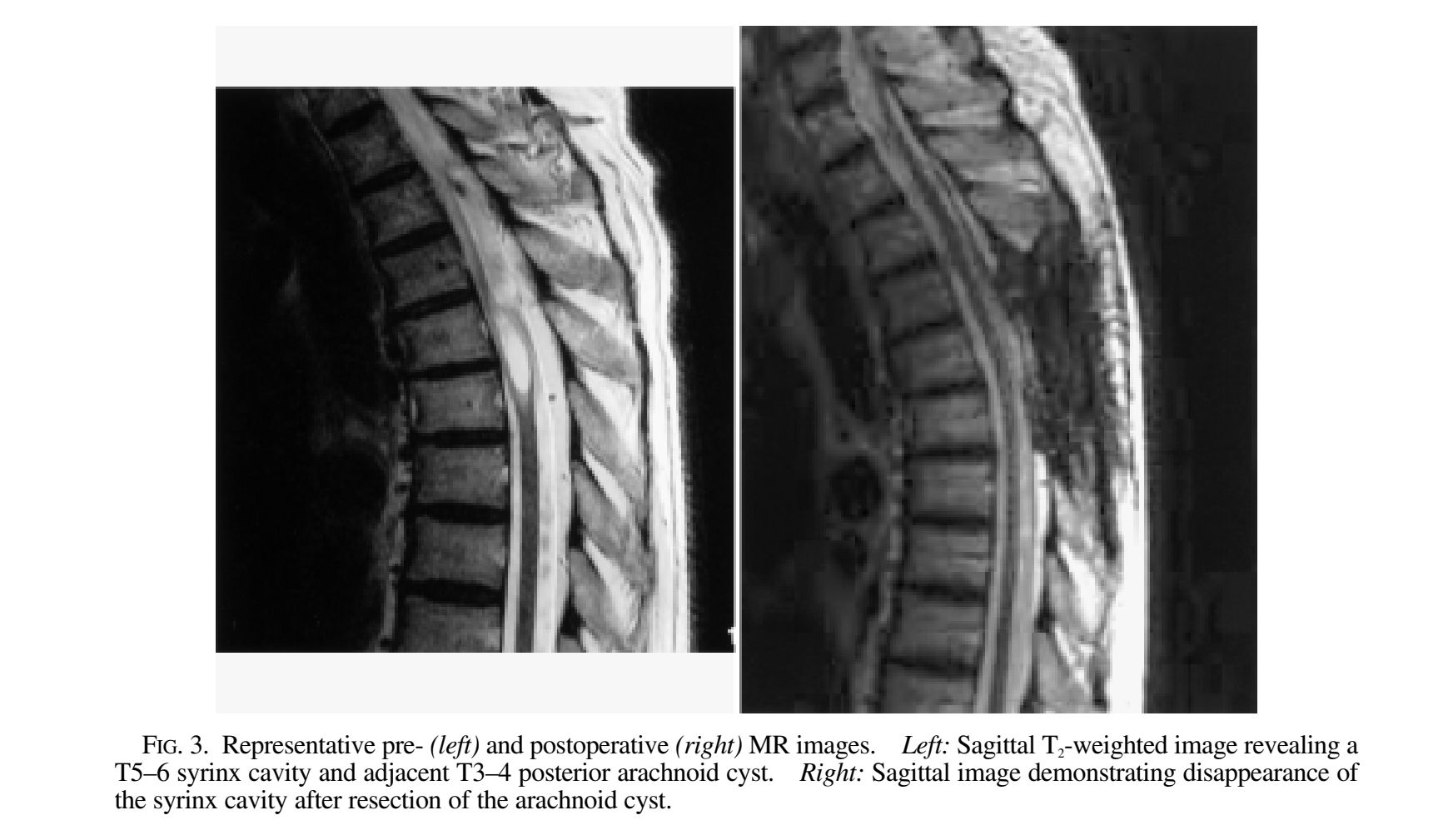




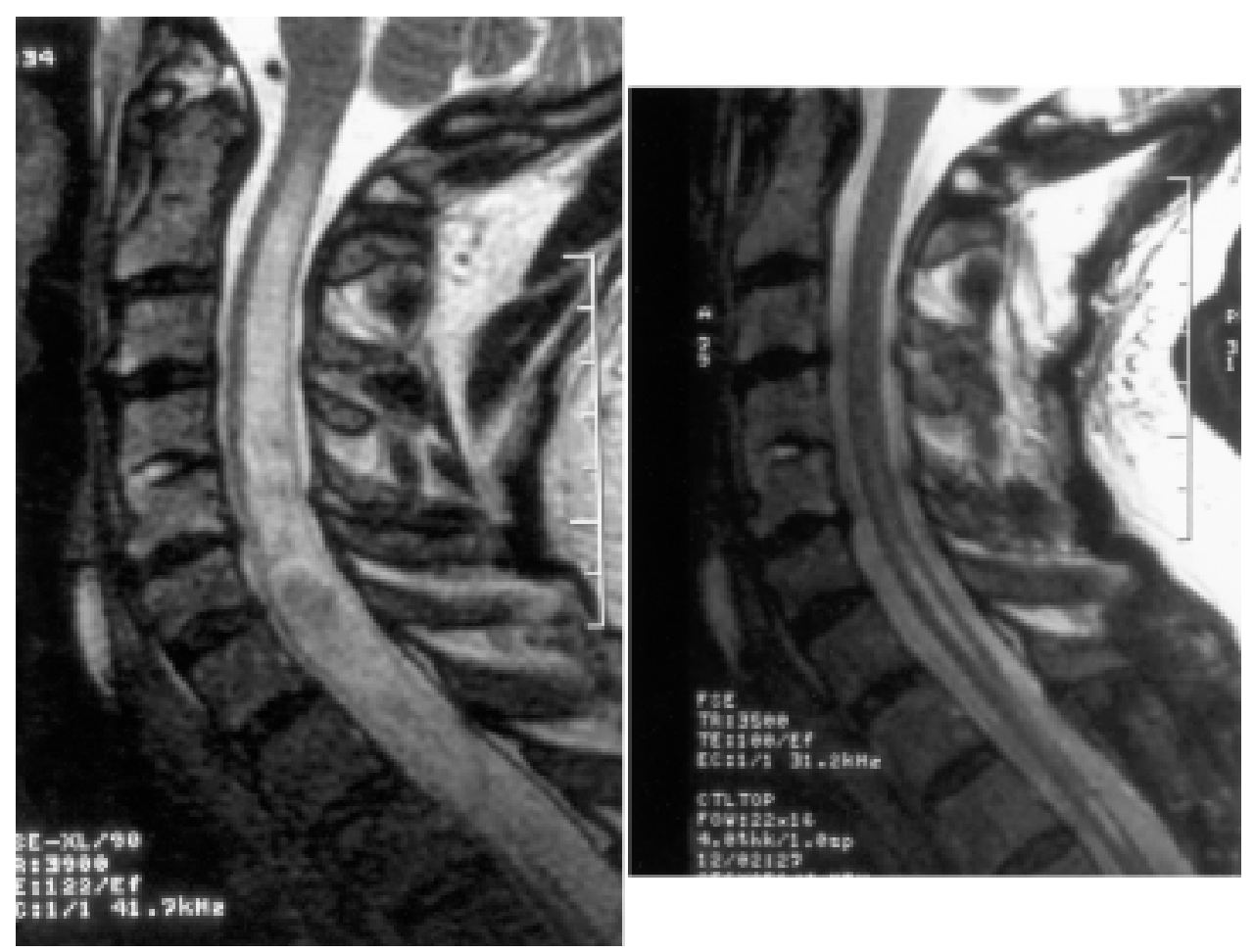

FIG. 4. Representative sagittal $\mathrm{T}_{2}$-weighted MR images revealing pre- (left) and postoperative (right) statuses in a patient in the present study. Left: Large C1-T1 syrinx cavity and a T2-3 arachnoid cyst. Right: The syrinx is significantly reduced in size after resection of the arachnoid cyst.

phenomenon is not associated with displacement of the neural elements or narrowing of the subarachnoid space.

Postmyelography CT is able both to confirm the presence of the cyst and to provide additional information regarding cyst morphology and filling characteristics. The injection can be performed via lumbar puncture, cisternal tap, or in some cases both. In some patients CT scanning will not readily demonstrate cyst opacification following the administration of a contrast medium in the lumbar region. Not infrequently large cysts obstruct the passage of the contrast agent injected via the lumbar route (that is, caudal to the cyst), thereby preventing immediate opacification in cysts that fill cephalad to the area of compression. In these cases, administration of contrast via a cisternal puncture or delaying the CT session $(\sim 12-24$ hours after injection of the dye) should allow demonstration of the filling of the cyst. It is also important for the radiologist to observe closely any subtle change in contrast agent flow in the area where an arachnoid cyst is suspected. In other cases, because CT myelography may not demonstrate any appreciable difference between the density of the contrast medium within the subarachnoid space and the presumed arachnoid cyst, it is nearly impossible to delineate the cyst walls, and the correct diagnosis of an arachnoid cyst can be easily missed. In this situation, the most obvious indication of the presence of a cyst is the flattening of the posterior aspect of the spinal cord, which is also commonly asymmetrically displaced within the spinal canal and may exhibit an abrupt alteration in its contour. The axial CT scans obtained caudal to the presumed cyst should show that the spinal cord has returned to its normal midline location and there should be no evidence of posterior flattening.

\section{Management of a Syrinx Cavity and an Adjacent Arachnoid Cyst}

There have been several previous reports in which the authors have described the association of syringomyelia with an adjacent arachnoid cyst. ${ }^{2,3,11,15}$ These reports are generally limited to case reports involving one or two patients, with Andrews, et al., ${ }^{2}$ reporting the largest series (five patients). In three of the patients in their series the lesions were associated with previous spinal surgery in the region of the cysts and in two the lesions were posttraumatic. All five patients underwent fenestration of the arachnoid cyst, one patient underwent placement of an arachnoid cyst shunt, and three patients underwent placement of a shunt in the syrinx cavity. To our knowledge, our series of eight patients is the largest reported in the literature to date. Moreover, our series represents a uniform patient population in which syringomyelia was associated with an arachnoid cyst and in which all patients underwent the same surgical procedure. Furthermore, resection of the arachnoid cyst and reestablishment of spinal subarachnoid CSF flow resulted in significant neurological improvement in each patient. Three-month postoperative imaging showed successful resection of the cyst and a marked decrease in the size of the syrinx cavity in all eight patients. Each patient underwent laminectomy in which the facet complex was preserved. Although the follow-up period has been relatively short, there have been no imag- 
ing or clinical signs of postoperative spinal deformity. A hemilaminotomy is also a therapeutic option in patients whose arachnoid cysts are predominantly unilateral in location. Presently, the placement of a syrinx cavity or arachnoid cyst shunt does not appear to have a role in the primary treatment of this entity.

\section{Conclusions}

Syrinx cavities can be associated with arachnoid cysts, and these cysts are not likely to be an incidental finding, but frequently contribute to the filling mechanism of the syrinx via subarachnoid CSF flow obstruction. Primary surgical management should be directed toward removal of the cyst wall and restoration of normal CSF flow. Based on our experience we find that neurological recovery and significant reduction in syrinx cavity size are likely to occur after surgery.

\section{References}

1. Abou-Fakhr FS, Kanaan SV, Youness FM, Hourani MH, Haddad MC: Thoracic spinal intradural arachnoid cyst: report of two cases and review of the literature. Eur Radiol 12: 877-882, 2002

2. Andrews BT, Weinstein PR, Rosenblum ML, Barbaro N: Intradural arachnoid cysts of the spinal canal associated with intramedullary cysts. J Neurosurg 68:544-549, 1988

3. Bruneau M, Duprez T, Rommel D, Raftopoulos C: Surgical treatment of a syringomyelia associated with an idiopathic arachnoid malformation disclosed by preoperative MRI. Surg Neurol 62:552-555, 2004

4. Klekamp J, Batzdorf U, Samii M, Bothe HW: The surgical treatment of Chiari I malformation. Acta Neurochir (Wien) 138:788-801, 1996

5. Klekamp J, Batzdorf U, Samii M, Bothe HW: Treatment of syringomyelia associated with arachnoid scarring caused by arachnoiditis or trauma. J Neurosurg 86:223-240, 1997
6. Kramer KM, Levine AM: Posttraumatic syringomyelia: a review of 21 cases. Clin Orthop Relat Res 334:190-199, 1997

7. Kumar K, Malik S, Schulte PA: Symptomatic spinal arachnoid cysts: report of two cases with review of the literature. Spine 28:E25-E29, 2003

8. La Haye P, Batzdorf U: Posttraumatic syringomyelia. West J Med 148:657-663, 1988

9. Levi AD, Sonntag VK: Management of posttraumatic syringomyelia using an expansile duraplasty. A case report. Spine 23: 128-132, 1998

10. Osenbach RK, Godersky JC, Traynelis VC, Schelper RD: Intradural extramedullary cysts of the spinal canal: clinical presentation, radiographic diagnosis, and surgical management. Neurosurgery 30:35-42, 1992

11. Paramore CG: Dorsal arachnoid web with spinal cord compression: variant of arachnoid cyst? Report of two cases. J Neurosurg 93 (2 Suppl):287-290, 2000

12. Perret G, Green D, Keller J: Diagnosis and treatment of intradural arachnoid cysts of the thoracic spine. Radiology 79: 425-429, 1962

13. Perrouin-Verbe B, Lenne-Aurier K, Robert R, Auffray-Calvier E, Richard I, Mauduyt de la Greve I, et al: Post-traumatic syringomyelia and post-traumatic spinal canal stenosis: a direct relationship: review of 75 patients with a spinal cord injury. Spinal Cord 36:137-143, 1998

14. Silbergleit R, Brunberg JA, Patel SC, Mehta BA, Aravapalli SR: Imaging of spinal intradural arachnoid cysts: MRI, myelography, and CT. Neuroradiology 40:664-668, 1998

15. Takeuchi A, Miyamoto K, Sugiyama S, Saitou M, Hosoe H, Shimizu K: Spinal arachnoid cysts associated with syringomyelia: report of two cases and a review of the literature. J Spinal Disord Tech 16:207-211, 2003

Manuscript received November 23, 2005

Accepted in final form May 8, 2006.

Address reprint requests to: Ulrich Batzdorf, M.D., Division of Neurosurgery, David Geffen School of Medicine at UCLA, Center for Health Sciences, Room 74-145, 10833 Le Conte Avenue, Los Angeles, California 90095. email: ubatzdorf@mednet.ucla.edu. 